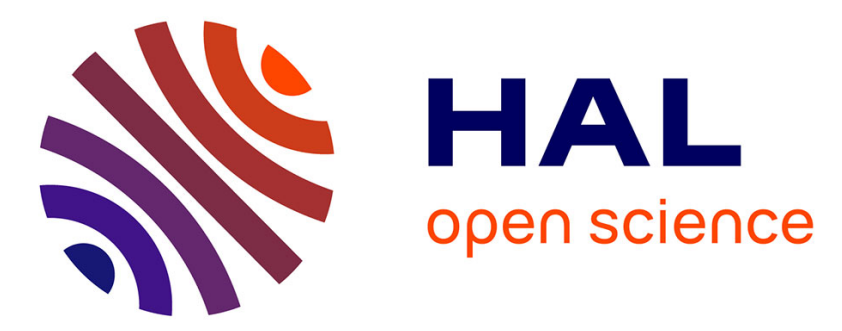

\title{
Recension: Juliette SIBON, Les Juifs de Marseille au XIVe siècle
}

\author{
Elodie Attia
}

\section{To cite this version:}

Elodie Attia. Recension: Juliette SIBON, Les Juifs de Marseille au XIVe siècle. 2013, pp.418-419. 10.3917/etr.0883.0401 . hal-01434138

\section{HAL Id: hal-01434138 \\ https://hal-amu.archives-ouvertes.fr/hal-01434138}

Submitted on 13 Jan 2017

HAL is a multi-disciplinary open access archive for the deposit and dissemination of scientific research documents, whether they are published or not. The documents may come from teaching and research institutions in France or abroad, or from public or private research centers.
L'archive ouverte pluridisciplinaire HAL, est destinée au dépôt et à la diffusion de documents scientifiques de niveau recherche, publiés ou non, émanant des établissements d'enseignement et de recherche français ou étrangers, des laboratoires publics ou privés.

\section{(1) (1) $\$$}

Distributed under a Creative Commons Attribution - NonCommercial - NoDerivatives| 4.0 


\title{
PARMI LES LIVRES
}

\author{
Institut protestant de théologie | «Études théologiques et religieuses »
}

2013/3 Tome 88 | pages 401 à 435

ISSN 0014-2239

Article disponible en ligne à l'adresse :

http://www.cairn.info/revue-etudes-theologiques-et-religieuses-2013-3-page-401.htm

\section{Pour citer cet article :}

«Parmi les livres », Études théologiques et religieuses 2013/3 (Tome 88), p. 401-435.

DOI 10.3917/etr.0883.0401

Distribution électronique Cairn.info pour Institut protestant de théologie.

(c) Institut protestant de théologie. Tous droits réservés pour tous pays.

La reproduction ou représentation de cet article, notamment par photocopie, n'est autorisée que dans les limites des conditions générales d'utilisation du site ou, le cas échéant, des conditions générales de la licence souscrite par votre établissement. Toute autre reproduction ou représentation, en tout ou partie, sous quelque forme et de quelque manière que ce soit, est interdite sauf accord préalable et écrit de l'éditeur, en dehors des cas prévus par la législation en vigueur en France. Il est précisé que son stockage dans une base de données est également interdit. 
le «secret» de leur amour), et les sermons prononcés, sauf rares exceptions, à Saint-Nicolas (il en analyse les contenus).

Espérons que A. donnera une suite à ce livre et étudiera, avec la même science et la même finesse, les périodes suivantes de la vie de Schweitzer, en particulier les années entre la fin de la Première Guerre mondiale et son retour à Lambaréné, sur lesquelles nous avons peu de choses.

André GOUNELLE

\section{ÉTUDES JUIVES}

Juliette SiBon, Les Juifs de Marseille au $X I V^{e}$ siècle, Paris, Cerf, coll. « Nouvelle Gallia Judaïca 6 », 2011. $23,5 \mathrm{~cm} .585$ p. ISBN $978-2-204-$ 09506-8. € 48

L'ouvrage, préfacé par Henri BRESC, se présente comme une monographie sur les Juifs de Marseille élaborée à partir des registres latins de notaires marseillais datés du XIV ${ }^{\mathrm{e}}$ siècle déposés à la Bibliothèque nationale de France. Il élargit les problématiques bien connues de l'histoire économique et sociale pour s'orienter vers l'histoire culturelle des élites juives marseillaises. Ces documents de la pratique témoignent d'activités réelles qui dépassent leurs interdictions théoriques (tel le mutuum et les charges publiques). En trois scansions d'égale importance, Juliette SIBON propose d'explorer l'assise matérielle, l'identité et, enfin, malgré certaines lacunes des sources, la culture savante des notables Juifs de Marseille. En début de première partie, elle éclaire le rapport des Juifs à l'usure sous de nouvelles perspectives (chap. 1). Les Juifs marseillais jouent un rôle pragmatique aux côtés d'autres créanciers chrétiens, et l'étude donne une image détaillée des prêteurs et des clients chrétiens, dont les plus nombreux sont les laboureurs et les artisans (p. 48) validant le prêt juif de petite somme à rayon court. Mais, comme en Cerdagne bien étudiée par C. Denjean, un réseau en amont du crédit juif tissé avec des capitalistes chrétiens préexiste et favorise la solidarité entre élites. Les Juifs entrepreneurs et commerçants (chap. 2) sont difficiles à saisir sauf dans le cas des artisans (parfois entrepreneurs) « corailleurs » et des «tailleurs ». Certaines fonctions publiques, tel le courtage parfois effectué par des femmes, étaient officiellement reconnues par la justice angevine, dans l'ensemble plutôt bienveillante envers ses Juifs. avec Enfin, des entreprises de commercialisation montrent que les élites juives maintiennent leur position dans la vie économique du port. L'investissement dans l'immobilier et le foncier (chap. 3) confirme l'intégration économique et sociale, la confiance en l'existence dans le monde latin, la volonté d'investir dans l'exploitation agricole (vin) étant liée au commerce avec la Méditerranée occidentale. En deuxième partie, l'auteur soulève la question de l'identité des notables juifs (chap. 4). L'archaïsme dans le choix des noms personnels souligne la différenciation volontaire d'avec la société majoritaire, tandis que la transmission du patronyme fait ressortir un certain conformisme socioculturel avec le patriciat urbain marseillais. La question des doubles noms (p. 239) souligne une difficulté permanente pour identifier les individus entre sources hébraïques et latines. Quant aux stratégies matrimoniales et successorales (chap. 5), S. s'interroge sur la taille et la composition des familles, leurs conflits internes, observant les testaments (4 documents sont analysés) et les contrats de mariage 
(rares en latin car ils dépendent du droit rabbinique, donc rédigés en hébreu) ainsi que la place des femmes dans la famille. Enfin, le rôle du citoyen juif, son implication dans la cité et l'organisation interne des communautés dirigées par une poignée d'individus (chap. 6) sont décrits dans les grandes lignes faute de sources suffisantes. L'autonomie du Qahal n'était pas signe de fermeture, même si le pouvoir des dirigeants sur leurs coreligionnaires semblait total et coercitif (herem). En dernière partie, $\mathrm{S}$. cherche à retracer le milieu culturel et savant juif marseillais (chap. 7) mais les sources semblent le rétrécir à celui des médecins - du fait de leur surreprésentation avérée dans lesdites sources. Ils semblent aussi nombreux que les grands prêteurs et les conditions de transmission de leur savoir sont liées à des dynasties médicales, à leurs bibliothèques (étonnamment invisibles ici) ou à un apprentissage. La pratique médicale est décrite en détail, tant pour les « médecins physiciens » que pour les « chirurgiens ». Puis, la question de l'attachement à la pratique religieuse de ces élites est posée (chap. 8). Cet attachement, érodé théoriquement suite à l'expansion de l'averroïsme, est analysé ici par le biais de deux trousseaux et d'une série de litiges. À Marseille, le mobilier, le luminaire et les ustensiles de cuisine donnent des indices clairs d'une pratique religieuse effective, de même que l'activité de boucherie, le respect strict du calendrier juif. Les litiges jugés par la cour angevine ne semblent pas traduire de rejet spécifique. Plutôt marginal dans les sources marseillaises, l'antisémitisme médiéval et l'antijudaïsme sont néanmoins abordés au travers de quelques exemples (p. 454). Pour finir, la question des Néophytes (chap. 9) est naturellement développée par comparaison avec les études de M. Kriegel et D. Iancu-Agou. S. présente le statut des nouveaux convertis, leur ascension sociale, et la protection des élites chrétiennes marchandes et puissantes offerte aux convertis. Ces derniers semblent d'ailleurs garder de bons contacts avec la minorité juive, en dehors de la sphère économique. Pour finir, un fichier prosopographique, des transcriptions latines et des index complètent ce livre, désormais incontournable, consacré aux Juifs de Marseille.

Élodie ATTIA

Jean BAUMGarten, Julien DARMON, dir., A ux origines $d u$ judaïsme, Paris, Les Liens qui Libèrent/Actes Sud, 2012. 24,5 cm. 525 p. 95 ill. ISBN 979-10209-0003-6. € 38 .

L'ouvrage collectif dirigé par Jean BAUMGARTEN et Julien DARMON doit être salué comme une contribution importante à la diffusion des études juives en France. Il se donne comme objectif de " dépasser l'aporie qui consisterait à définir l'histoire juive comme l'histoire des Juifs et les Juifs comme ceux qui participent de l'histoire juive» (p. 9). Pour ce faire, il s'attache à présenter avant tout les productions intellectuelles et culturelles juives, perçues comme «le lieu premier d'une histoire qui n'a, durant des siècles, pas pu s'incarner dans une geste "nationale" ", une histoire qui serait avant tout celle de la " révélation progressive de la nature spirituelle du monde » (p. 10). C'est pourquoi le livre est construit en deux parties, l'une qui porte sur les œuvres qui structurent la vie religieuse et intellectuelle juive, et l'autre qui aborde l'histoire sociale, celle des communautés et des migrations, de la confrontation avec la modernité et de la création de l'État d'Israël. Cette façon d'aborder l'histoire juive restitue au mieux la perspective 